\title{
Revisão de literatura da punição corporal
}

\author{
Olivia Justen Brandenburg \\ Lidia Natalia Dobrianskyj Weber \\ Universidade Federal do Paraná
}

\begin{abstract}
RESUMO
Foi desenvolvida uma pesquisa documental com o objetivo de revisar a literatura científica e levantar os dados que têm sido encontrados em pesquisas sobre Punição Corporal (PC) normativa. Amostra: 90 resumos de pesquisas realizadas nos últimos 13 anos (1990 a 2002). Tais pesquisas foram selecionadas na base de dados do PsycINFO. A análise documental qualitativa com análise de conteúdo dos resultados. Os principais resultados encontrados foram: 1) alta incidência da PC; 2) a opinião sobre o uso da PC variou de acordo com diversos fatores; 3) fatores situacionais, sociodemográficos, culturais e religiosos influenciam no uso da PC; 4) a PC normativa traz efeitos negativos para os filhos; 5) a PC tende a ser transmitida intergeracionalmente; 6) a PC é um risco para maltrato. A análise dos dados permitiu discutir o uso da PC e chamar a atenção da comunidade científica para a importância do estudo deste assunto.
\end{abstract}

Palavras-chaves: revisão de literatura; punição corporal; disciplina.

\begin{abstract}
Review of literature on corporal punishment

Documental research was undertaken with the purpose of reviewing scientific literature and obtaining data found in studies on normative Corporal Punishment (CP). Sample: 90 abstracts of studies undertaken in the last 13 years (1990 to 2002). These studies were selected from the PsycINFO database. Qualitative documental analysis was carried out on the contents of the results. The principal results encountered were: 1) high incidence of $\mathrm{CP}$; 2) variation of opinions on the use of $\mathrm{CP}$ depending on a diversity of factors; 3) situational, socio-demographic, cultural and religious factors influence the use of $\mathrm{CP}$; 4) normative $\mathrm{CP}$ has negative effect on ones children; 5) $\mathrm{CP}$ tends to be passed on from generation to generation; 6) CP represents a risk of mistreatment. The analysis of the data enabled the discussion of the use of $\mathrm{CP}$ as well as to draw the attention of the scientific community to the importance of studying this subject.
\end{abstract}

Keywords: literature review; corporal punishment; discipline.

Uma definição muito utilizada na literatura para punição corporal é a de Straus (1994): "punição corporal é o uso de força física com a intenção de fazer a criança experimentar dor, mas sem machucá-la, com a finalidade de correção ou controle do comportamento da criança". No presente trabalho, o termo "punição corporal" será utilizado para designar o ato de pais baterem em seus filhos, crianças ou adolescentes, como um método de disciplina. A punição corporal será tratada como punição normativa, não incluindo neste termo a noção do maltrato.

A Análise do Comportamento traz argumentos contra o uso da punição. Skinner (1976) mostrava-se totalmente contrário ao uso da punição, descrevendo claramente os subprodutos nocivos desta. O efeito imediato da punição física é suficiente para as pessoas continuarem a utilizar esta prática educativa, mas geralmente, depois de um tempo sem a punição, o comportamento reaparece. Além disso, o uso das punições pode levar ao fenômeno do "desamparo aprendido" (Seligman, 1977). O desamparo aprendido é a incapacidade de aprendizagem de novas respostas de esquiva diante de uma história de exposição a estímulos aversivos não contingente a qualquer resposta. $\mathrm{Se}$ as punições, especialmente físicas, não são contingentes e dependem do humor dos pais, pode ocorrer o desamparo.

Skinner (1976) mencionava, como um aspecto nocivo da punição, os comportamentos emocionais (choro, medo, ansiedade, raiva), os quais podem ser condicionados a aparecerem em outras ocasiões não punitivas. Também pode haver o condicionamento de comportamentos de fuga e esquiva (por meio do reforçamento negativo), que reduzem ou livram a criança da estimulação aversiva ou pré-aversiva (Sidman, 2001; Skinner, 1976). Além disso, na aplicação da punição corporal pode ocorrer uma associação entre a dor que a criança sente e o amor em relação aos seus pais. Este emparelhamento de estímulos pode ensinar a criança a usar o mesmo método em outras situações de sua vida ou ainda, suportar situações aversivas e disfuncionais que deveriam ser terminadas (Weber, 2001). 
Muitos pais batem em seus filhos. Uma pesquisa em Curitiba encontrou que quase $90 \%$ das crianças e adolescentes apanhavam, principalmente dos pais (Weber, Viezzer e Brandenburg, 2004). Bater nos filhos é correto? Enquanto na análise do comportamento prevalece uma posição contra o uso de punição corporal, na psicologia, de forma geral, há muita polêmica. Certamente as pesquisas empíricas a respeito da punição corporal podem trazer esclarecimentos sobre o debate bater versus não bater nos filhos.

Houve dificuldade em encontrar material nacional sobre punição corporal normativa. No Brasil, pesquisadores têm se preocupado muito mais com a violência física infantil doméstica (Carswell, 1990; Camargo, 1996; Furlotti, 1999; Meneguel, Giugliani \& Falceto, 1998; Pascolat, 2001; Roque, 2001; Santos, 1999; Weber, Viezzer, Brandenburg \& Zocche, 2002) do que com as palmadas cotidianas e ocasionais, tidas como "normais" e necessárias. Assim, optou-se por uma revisão de literatura internacional, para investigar o quê e como autores internacionais têm pesquisado sobre este assunto.

Parke (2002) organizou a produção na área de punição corporal, propondo uma divisão em quatro fases: fase descritiva (entre anos 40 e 60), fase experimental (entre anos 60 e 70), fase de pesquisas sobre o efeito da punição corporal na criança (a partir dos anos 80), fase da politização da punição, incluindo uma valoração do uso da punição corporal. Larzelere e Johnson (1999) revisaram sete artigos sobre proibição de punição corporal na Suécia. Straus (2000) revisou nove artigos demonstrando que para prevenir a violência física contra a criança é necessário eliminar o uso da punição corporal. Andero e Stewart (2000) fizeram uma revisão de literatura sobre as razões que levam pais a utilizarem a punição corporal, enfatizando que este ponto é negligenciado pelos pesquisadores e deve ser mais enfocado. Larzelere (2000) revisou cinco anos de pesquisas que relacionavam a punição corporal normativa com efeitos na criança e encontrou problemas em razão de diferenças metodológicas. Gershoff (2002) encontrou prevalência de efeitos negativos nos filhos e propôs um modelo para explicar como a punição corporal pode afetar a criança ou adolescente.

Revisão de literatura é uma forma interessante de analisar um tema tão polêmico como o uso da punição corporal. O objetivo da presente pesquisa foi revisar a literatura científica sobre punição corporal normativa, enriquecendo a discussão e o debate, pensando principalmente em contribuir com a produção nacional sobre o assunto.

\section{MÉTODO}

\section{Amostra}

90 pesquisas empíricas sobre punição corporal normativa aplicada por pais em seus filhos, publicadas entre 1990 e 2002, disponíveis na base de dados online do Psychological Abstracts PsycINFO.

\section{Procedimento de seleção de resumos}

Foram utilizadas as palavras-chave: punição física (psysical punishment), palmada (spanking) e punição corporal (corporal punishment). Critérios de seleção: pesquisas empíricas, amostra de população normal, divulgadas em artigos científicos ou em dissertações, sobre punição corporal normativa aplicadas por pais na disciplina de seus filhos.

\section{Análise de dados}

Foi realizada uma análise documental dos resumos de pesquisas selecionados. Os resultados das pesquisas foram traduzidos para o português. A principal análise foi realizada com o conteúdo dos resultados das pesquisas selecionadas, agrupando-os em categorias. Observa-se que uma mesma pesquisa pode ter apresentado mais de um tipo de resultado e por isso foi classificada em mais de uma categoria. A análise realizada tem caráter de levantamento dos dados encontrados nas pesquisas, sem objetivo de comparar os métodos utilizados em tais pesquisas.

\section{RESULTADOS}

Dentre as 90 pesquisas selecionadas, $74(81 \%)$ estavam publicadas em periódicos, e 17 (19\%) eram dissertações. A maioria foi realizada nos EUA, apenas 21 (23\%) foram realizadas em outros países ou com amostras estrangeiras nos EUA.

As pesquisas selecionadas foram categorizadas de acordo com o conteúdo dos resultados oferecidos nos resumos, obtendo-se: 1) $14(11 \%)$ resultados referentes à incidência da punição corporal; 2) 15 (12\%), à opinião dos participantes sobre o uso deste método disciplinar; 3) 51 (41\%), aos fatores situacionais, sociodemográficos, culturais e religiosos; 4) 21 (17\%), aos "efeitos" da punição corporal normativa para os filhos; 5) 8 (6\%), à transmissão intergeracional desta prática; 6) 5 (4\%), ao risco de maltrato; 7$) 11$ (9\%), a outros aspectos relacionados com o assunto.

\section{1) Incidência da punição corporal}

As pesquisas mostraram que a maioria absoluta dos pais bate. Foram encontradas freqüências variando de 57\% até 94\% (Ateah \& Parking, 2002; Dietz, 2000; Barrish, 1996; Giles, Straus \& Sugarman, 1995; Graziano \& Namste, 1990; Holden, Coleman \& 
Schmidt, 1995; Straus \& Donnely, 1993; Straus \& Stewart, 1999). Pesquisadores relataram diferença na incidência da punição corporal de acordo com a severidade do método (Dietz, 2000) e de acordo com a combinação entre severidade e idade do filho (Straus \& Stewart, 1999). Das 14 pesquisas nesta categoria, 6 delas foram realizadas com população estrangeira: no Caribe (Gopaul-Mcnicol, 1999), no norte da Irlanda (Murphy-Cowan \& Stringer, 1999) e na Jordânia (Taleb, 2001), as porcentagens apresentadas foram mais altas; enquanto entre afro-americanos (Hopkins, 1998), no Egito (Yousser, Attia \& Kamel, 1998) e na Dinamarca (Christensen, 2001) as porcentagens foram mais baixas.

\section{2) Opinião dos participantes em relação ao uso da punição corporal por pais}

A categoria 2 agrupou resultados de pesquisas sobre como as pessoas avaliam a prática disciplinar de bater nos filhos. Nessa categoria apareceram pesquisas em que adultos apoiaram com maior (Flynn, 1994) ou menor (Ateah \& Parking, 2002) frequiência o uso da punição física. Adolescentes ou universitários achavam que bater em filhos era mais apropriado quando fosse palmada menos severa e quando a criança merecia (Kelder, Namara, Carlson \& Lynn, 1991), quando o filho era de idade pré-escolar e quando desrespeitava ou violava normas estabelecidas (Flynn, 1998); além disso, os adolescentes que apanharam quando pequenos apoiaram mais esta prática do que aqueles que não apanharam (Graziano \& Namste, 1990; Rabin, 1997). Na Nova Zelândia, adolescentes escolheram a punição física como última opção entre as alternativas (Blampied \& Kahan, 1992). Apenas uma pesquisa foi feita sobre a opinião de crianças, as quais foram, de forma geral, favoráveis ao seu uso (Catron \& Masters, 1993).

Nas pesquisas em que foi investigada a opinião sobre a eficiência e os efeitos da punição corporal, encontrou-se que, apesar de os adultos acreditarem ser o diálogo um método melhor do que a punição corporal, não traz efeitos negativos quando é usada ocasionalmente (Furnham, 1995); além disso, o uso da punição corporal foi caracterizado com pontos positivos por pais (Gough \& Reavey, 1997) e por idosos afroamericanos (Mosby, Rawls, Meehan, Mays \& Pettinari, 1999).

Foram encontradas quatro pesquisas de opinião com diferentes profissionais: numa amostra de profissionais afro-americanos não especificados, a maioria apoiou o uso da punição corporal (Hopkins, 1998); os assistentes sociais favoráveis ao uso deste método disciplinar demonstraram menor percepção de maustratos contra a criança e menor probabilidade de denúncia do agressor (Ashton, 2001; Mardis, 2001); a maioria dos psicólogos era contra o uso de punição corporal apesar de muitos recomendarem e muitos utilizarem este método com seus filhos. $\mathrm{O}$ apoio deste método variou de acordo com a experiência prévia de vida do psicólogo e de acordo com a linha teórica: a maioria dos psicólogos baseados no humanismo e na teoria familiar sistêmica era favorável, enquanto a maioria dos behavioristas e behavioristas cognitivos apresentou-se contra (Kaplan, 1996).

\section{3) Fatores situacionais, sociodemográficos, culturais e religiosos}

A categoria 3 reuniu os resultados que diziam respeito a dois grandes fatores: a) situacionais, como características dos pais e o momento em que eles batem; e b) sociodemográficos, culturais e religiosos, como a localidade, a cultura e a religião em que os pais estão inseridos, sexo, idade, nível de educação, empregabilidade etc.Todos estes são fatores que, de alguma forma, estão predispondo os pais a baterem em seus filhos e são chamados por alguns autores de "preditores".

A respeito dos fatores situacionais, muitos resultados apresentados demonstraram que características dos pais podem predizer o uso da punição corporal normativa. Pesquisas relacionaram o uso da punição corporal com irritação dos pais (Graziano \& Namste, 1990); raiva (Ateah, 2001; Watson, 2002), mauhumor (Holden \& cols., 1995), estresse (Dietz, 2000; Wade, 2000), estresse aliado à crença neste método (Crouch \& Behl, 2001), depressão (Wissow, 2001), saúde mental (Nobes \& Smith, 2002, Christensen, 2001), doença e infelicidade (Taleb, 2001), o objetivo dos pais na educação dos seus filhos (Christensen, 2001; Frias-Armenta, 1999).

As pesquisas mostraram que pais bateram em seus filhos quando estes apresentaram baixo desempenho escolar, desafiaram os pais e fugiram de casa (Vargas, Lopez, Perez \& Zuniga, 1995) ou apresentaram comportamento agressivo (Holden \& cols., 1995); quando a mãe percebeu a intenção do mau comportamento da criança (Ateah, 2001). Uma pesquisa no Egito encontrou que comportamentos punidos corporalmente foram: desobediência, mentira, destruir pertences de outros, desrespeitar os pais, fugir de casa, baixo rendimento escolar (Yousser \& cols., 1998); outra pesquisa na Jordânia encontrou que as mães que preferiam utilizar punição corporal tinham filhos com: busca de atenção contínua, medo da mãe, distúrbios de sono, isolamento social, pouco apetite, quebra e destruição de objetos e dificuldade de concentração (Taleb, 2001).

Em relação ao momento do dia em que pais fazem uso da punição corporal, uma pesquisa encontrou que mães utilizaram punição física para reduzir compor- 
tamentos inapropriados à idade especialmente no horário das refeições e em situações de aprendizagem (Culp, Culp, Dengler \& Maisano, 1999).

Pôde-se identificar nos resultados características do ambiente em que os pais estão inseridos. Quanto à composição familiar, foi encontrado maior uso de punição corporal em filhos que moram com ambos os pais (Straus \& Donnely, 1993), o que condiz com pesquisas realizadas na Inglaterra que encontraram similaridade na frequiência e na severidade no uso de punição corporal entre pais e mães (Nobes \& Smith, 1997; Nobes, Smith, Upton \& Heverin, 1999) e encontraram que mães solteiras não punem com maior freqüência nem com maior severidade que mães casadas (Nobes \& Smith, 2002). No contexto familiar, ainda, houve pesquisas que mostraram maior freqüência do uso da punição corporal em famílias com pais menos responsivos (Turner \& Finkelhor, 1996; Wissow, 2001), famílias que tinham avós morando junto e de quem a mãe precisava cuidar (Stolley e Szinivacz, 1997), ambiente familiar agressivo (Muller, 1996), famílias no Egito que moravam junto com estrangeiros e viviam em ambiente de brigas (Yousser \& cols., 1998), famílias na Nova Zelândia com violência entre o casal e com abuso sexual infantil (Woodward $\&$ Fergusson, 2002).

Outras pesquisas sobre fatores situacionais: a opinião de pessoas próximas não afetou a decisão das mães em utilizar este método (Morris, 1996); fontes de informação, como pessoas e livros, foram percebidas como recomendáveis para o uso da punição corporal por mães que faziam uso (Walsh, 2002); a posição favorável à punição corporal também contribuiu para o uso deste método disciplinar (Frias-Armenta, 1999; Holden \& cols., 1995; Wade, 2000).

A respeito do fator religião, muitas pesquisas compararam diferentes religiões e verificaram que punem corporalmente com maior frequiência pessoas que seguem uma religião com leitura literal da Bíblia (Grasmick, Bursik \& Kimpel, 1991; Wiehe, 1990); religião protestante (Ellison, Bartkowski \& Segal, 1996; Flynn, 1994; Hopkins, 1998; Gershoff, Miller \& Holden, 1999); pessoas que seguem religião mais conservadora no Norte da Irlanda (Murphy-Cowan \& Stringer, 2001). Psicólogos judeus apresentaram-se mais favoráveis ao uso de punição corporal do que psicólogos protestantes (Kaplan, 1996), e entre os profissionais afro-americanos, os de religião batista eram mais favoráveis do que católicos. Sem especificar qual religião, uma pesquisa encontrou que o fator religião afetou a opinião das pessoas sobre punição corporal (Rabin, 1997), e outra pesquisa mostrou que não existe esta relação (Furnham, 1995).

O uso da punição corporal foi comparado entre diferentes países: coreanos e japoneses apanhavam com menor frequiência que americanos (Englehart \& Hale, 1990); em ambos os grupos de americanos e indianos, a maioria relatou ter sido punida fisicamente na infância, e o maior recebimento de punição corporal na infância foi associado com maior aceitação deste método na vida adulta (Graziano, Lindquist, Kunce \& Munjal, 1992); a punição corporal foi mais deliberada entre pais no Caribe do que nos Estados Unidos (Gopaul-Mcnicol, 1999); tanto mães latinas como européias preferiram utilizar métodos disciplinares não físicos, mas as mães latinas relataram maior prevalência e frequiência da punição física (Wade, 2000). Uma pesquisa com imigrantes em Israel, mostrou que sujeitos originados de diferentes regiões apresentavam opiniões distintas sobre a adequação do uso de punição corporal (Shor, 1999). Além de comparações de um país com o outro, apareceram pesquisas comparando uma região do EUA com a outra: no Nordeste dos EUA houve menor aceitação da punição corporal e no Sul, maior (Flynn, 1994); os adolescentes do Nordeste tenderam a apanhar menos e a justificar menos a palmada do que os sujeitos do Sul (Flynn, 1996). Quando houve controle de afiliação religiosa, gênero e educação dos pais, a influência da região diminuiu em relação às experiências com punição física (Flynn, 1996).

Pesquisas mostraram que fatores sociodemográficos fizeram diferença quanto à opinião favorável ao uso da punição corporal e quanto à utilização desta prática. A opinião de pessoas a favor variou de acordo com diversos fatores: adolescentes negros se apresentaram mais favoráveis do que adolescentes brancos (Flynn, 1998); indivíduos com menor nível de educação apoiaram mais o uso da punição corporal (Ellison \& Sherkat, 1993; Flynn, 1994); menor valor de salário e menor o nível socioeconômico foram associados à maior aceitação desta prática (Flynn, 1994; Kaplan, 1996; Vargas \& cols., 1995); homens apoiaram mais o uso deste método do que mulheres (Flynn, 1998; Hopkins, 1998; Kaplan, 1996; Rabin, 1997); quanto à posição política, eleitores conservadores apresentaram mais opiniões a favor do que contra a punição corporal (Furnham, 1995).

Quando a utilização da punição corporal foi avaliada, pesquisas encontraram variações de acordo com alguns fatores relacionados aos pais: afro-americanos, comparados aos brancos, usam a punição corporal em crianças mais velhas, apresentam maior freqüência de punições mais severas (Hopkins, 1998); mães que usavam mais esta prática eram mais jovens (Taleb, 2001); a punição corporal prevaleceu entre pais com baixo nível socioeconômico (Dietz, 2000; Straus \& Stewart, 1999; Taleb, 2001; Vargas \& cols., 1995), o desemprego dos pais esteve relacionado ao maior uso deste método (Jackson, Gyamfi, Brooks \& Blake, 1998; Taleb, 2001). Alguns resumos de pesquisas 
(Bradley, Corwyn, Burchinal, Pipes-Mcdoo \& GarciaColl, 2001; Giles \& cols., 1995; Rabin,1997) indicaram que variáveis como idade, gênero, raça, nível socioeconômico e religião se relacionaram com uso de punição corporal, mas estas relações não foram especificadas.

Uma única pesquisa encontrou que os fatores de gênero e idade não influenciaram a opinião das pessoas sobre a punição corporal (Furnham, 1995), e duas pesquisas encontraram que não interferiram no uso da punição corporal o nível de educação (Morris, 1996) e o gênero do filho (Saffer, 1999).

\section{4) Conseqüências da punição corporal para os filhos}

A maioria das pesquisas dessa categoria encontrou associação da punição corporal com algum aspecto do filho, criança ou adolescente. Correlações negativas apareceram entre uso da punição corporal e ajustamento psicológico (DeVet, 1997; Rohner, Kean \& Cournoyer, 1991); desempenho acadêmico de adolescentes sul-africanos (Cherian, 1994); autoconceito (Barrish, 1996); relacionamento entre pais e adolescentes (De Vet, 1997); e bem-estar geral (Bachar, Canetti, Bonne, Denour \& Shalev, 1997). Correlações positivas foram encontradas entre uso da punição corporal e posteriores sintomas depressivos, pensamentos suicidas, abuso de álcool e violência contra esposa (Straus \& Kantor, 1994); agressividade com os colegas (Strassberg, Dodge, Pettit \& Bates, 1994); angústia e depressão (Turner \& Finkelhor, 1996); sintomas psiquiátricos (Backar \& cols., 1997); tendência de se engajar em comportamentos anti-sociais e de agir impulsivamente (Straus \& Mouradian, 1998); envolvimento com brigas (Simons, Lin \& Gordon, 1998); crueldades com animais na infância ou na adolescência (Flynn, 1999); psicopatologias (Spencer, 1999); suicídio, depressão e violência contra parceiro na vida adulta (Good, 1999); problemas socioemocionais das crianças (Eamon \& Zuehl, 2001); depressão, ansiedade, uso de álcool, comportamento anti-social (FriasArmenta, 2002); ansiedade, depressão, comportamento anti-social, dificuldades em relações com pares nas crianças afro-americanas e nas euro-americanas (Matta, 2002).

Uma pesquisa encontrou associação entre punição corporal e problemas de comportamento de crianças afro-americanas, nas comunidades onde este método disciplinar era pouco usado (Simons, Lim, Gordon, Brody, Murry \& Conger, 2002). Outra pesquisa verificou um aumento de problemas de comportamento em crianças euro-americanas, afro-americanas e hispânicas, ao longo do tempo, no contexto de pouco apoio emocional (McLoyd \& Smith, 2002), o que condiz com o fato de a punição corporal não se relacio- nar com agressividade, delinqüência e bem-estar psicológico das crianças quando controlada a variável envolvimento parental (Simons, Johnson \& Conger, 1994). Ainda outra pesquisa comprovou o modelo de aprendizagem social, em que a punição corporal influencia comportamento agressivo de crianças, e não o modelo da teoria do temperamento, em que a agressividade da criança influencia o uso de punição corporal pelos pais (Muller, Hunter \& Stollak, 1995). Em uma pesquisa, os participantes afirmaram que a punição corporal recebida quando criança teve impacto positivo na vida adulta (Spencer, 1999).

\section{5) Transmissão intergeracional da punição corporal}

Dos resultados encontrados nessa categoria, um apresentou que a experiência com punição corporal na infância não influenciou a posição e o uso deste método (Hopkins, 1998), enquanto outros sustentaram o contrário, que adultos tendem a bater em seus filhos quando apanharam na infância (Dietz, 2000; FriasArmenta, 2002; Mosby e cols,, 1999; Murphy-Cowan \& Stringer, 1999; Saffer, 1999; Taleb, 2001). Sherman (1997) apresentou dados sobre os fatores que levaram pessoas com experiência de palmada a continuarem a usar a punição corporal com seus filhos ou mudarem o padrão; encontrou que pouco mais da metade mudou influenciada por alguns fatores: satisfação com o seu papel de pais, influência negativa de ter apanhado quando criança, aumento na severidade da punição recebida quando criança em casa, aumento na frequiência da punição corporal recebida fora de casa e relação negativa com a mãe e com o pai.

\section{6) Risco que a punição corporal representa para o maltrato físico}

Esta categoria reuniu os resultados referentes ao continuum entre punição corporal normativa e abusiva. Em duas pesquisas a punição corporal acabou em ferimentos mais graves, com caráter de violência física (Graziano \& Namste, 1990; Yousser \& cols., 1998), e em outra pesquisa encontrou-se que o aumento da frequiência pode estar acompanhado do aumento da severidade da punição corporal (Hopkins, 1998). Kaplan (1996) encontrou que, na opinião de mais da metade de psicólogos, qualquer punição corporal é um maltrato físico, mas para muitas pessoas o limite entre punição corporal normativa e abusiva não é claro (Frias-Armenta, 1999).

Outros resultados não se encaixaram em nenhuma categoria das já especificadas, por isso foram agrupadas numa última categoria sem que apresentassem um ponto em comum. Três pesquisas encontraram que grupos educativos para pais podem diminuir o uso da punição corporal (Hopkins, 1998; Nicholson, Anderson, 
Fox \& Brenner, 2002; Rabin, 1997). Duas pesquisas trouxeram dados sobre a interdição do uso da punição corporal na Suécia, mostrando mudanças desde que a lei entrou em vigor, como a diminuição do apoio público à punição corporal e diminuição do envolvimento dos adolescentes com crime, álcool, drogas e suicídio diminuíram (Durrant, 2000; Durrant, Broberg $\&$ Rose, 1999). Ainda houve duas pesquisas que fizeram experimentos demonstrando como a punição corporal é um fator estressor, tanto para pais quanto para filhos (Tschoban, 2001; Weitz, 2001), e duas pesquisas que encontraram fatores de mudança no comportamento das mães frente à reação negativa dos filhos à punição corporal (Holden, Thompson, Zambardo \& Marshall, 1997) e à mudança de valores (Davis, 1999).

\section{DISCUSSÃO}

\section{Entre a punição corporal e o maltrato}

O presente trabalho teve o cuidado de separar as pesquisas que tratassem exclusivamente do maltrato infantil. No entanto, dentre as pesquisas selecionadas há muitas que apresentaram dados misturados dos dois tipos de punição corporal. Estas pesquisas foram mantidas na análise justamente para ressaltar que o limite entre estes dois conceitos supostamente diferentes é, no mínimo, sutil.

Straus (1994); Longo, Azevedo e Guerra (2002) e Weber e cols. (2002) defendem a idéia de que há um continuum da palmada para o maltrato corporal contra a criança ou adolescente. Na presente pesquisa, os resultados analisados (Graziano \& Namste, 1990; Yousser \& cols., 1998) demonstraram justamente como a punição corporal aplicada para disciplinar pode ultrapassar o limite de dor da definição de punição corporal normativa, e acabar machucando e deixando marcas no corpo. Se na definição de punição corporal fica claro que os pais visam causar "dor sem machucar" para corrigir ou controlar um comportamento (Straus, 1994), na prática esse limite entre maior ou menor dor, maior ou menor machucado é questionável. Como Longo e cols. (2002) questionam: Quem pode medir o quanto dói?

Nesta linha de raciocínio questiona-se como as pesquisas determinam o quão severa é uma punição corporal. Em algumas pesquisas da categoria 1, apareceram diferenças na incidência de punição corporal normativa e de punição corporal severa (Dietz, 2000), havendo também diferença com idade dos filhos, os mais velhos receberam punições corporais mais severas (Straus \& Stewart, 1999). Questiona-se como avaliar quantitativamente o limite entre severo e nãosevero.
Um agravante do limite entre punição corporal normativa e abusiva é o fato de que as pesquisas analisadas demonstraram que, no monento em que a punição corporal ocorre, os pais estão alterados emocionalmente. Nos resultados da categoria 3, percebe-se como a irritação, a raiva, o mau-humor e o estresse são preditores do uso da punição física. Sabendo, segundo a Análise do Comportamento, que as emoções alteram a probabilidade de ocorrência de um comportamento, não é difícil imaginar como uma palmada, dita "inofensiva", pode num dia, em que o pai ou a mãe estejam alterados emocionalmente, se tornar uma violência física.

Cornet (1997) afirma que os pais descontam nos filhos suas irritações presentes ou mesmo passadas (pais que apanharam na infância) e tornam a agressividade um círculo vicioso. Se há o condicionamento de respostas de infligir dano com a emoção de raiva, isto aumenta ainda mais a probabilidade de eles baterem forte por apenas um pequeno comportamento inadequado da criança (Skinner, 1976). Neste caso, a punição corporal deixa de ter um caráter educativo, para transformar-se realmente em falta de autocontrole dos pais e levar à agressão.

Portanto, deve-se refletir sobre o fato de que usualmente costuma-se dividir "os pais que maltratam", do restante, como se fossem de naturezas diferentes. A justificativa mais freqüente dos que são favoráveis à punição é que uma palmada é muito diferente de um espancamento. No entanto, a palmada é a parte inicial de uma escala cuja natureza e princípios são os mesmos de um espancamento. Bater de leve ou dar uma surra são atitudes que seguem um mesmo princípio e não é possível delimitar onde termina um e começa o outro. Tanto que a prevenção para a violência física contra a criança é defendida por Straus (2002) no sentido de eliminar o uso da punição corporal. Quem dá um tapa será capaz de dar um tapa um pouco mais forte se o primeiro não resolver, pois fazem parte de contingências coercitivas da mesma forma. Comprovando este argumento, Weber, Viezzer e Brandenburg (2004) encontraram que, quanto mais freqüente era a punição corporal, mais forte era sua aplicação na criança.

\section{O que leva os pais a baterem em seus filhos?}

Os resultados sobre incidência demonstraram que a punição corporal é muito utilizada para educação dos filhos nos EUA e em outros países. Diante dessa disseminação do uso da punição corporal, levanta-se a questão sobre o que predispõe os pais a utilizarem este método disciplinar.

Esta pergunta pode ser respondida em parte pelos dados analisados na categoria 3. Os diversos fatores situacionais, sociodemográficos, culturais e religiosos 
citados, são apontados pelos pesquisadores como preditores do uso de punição corporal. Assim, o perfil de uma criança com menos idade, morando num ambiente familiar agressivo e disfuncional, numa cultura onde bater é mais comum, tem maior probabilidade de apanhar de seus pais. O perfil de mães com menos idade, níveis socioeconômico e educacional baixos; ou pais e mães que seguem religiões mais conservadoras ou protestantes, são perfis de pais que têm maior probabilidade de utilizar a punição corporal. A importância de se estudar e de se avaliar esses fatores é de que são pontos que indicam onde atuar para prevenir o uso de punição corporal e do maltrato, já que o segundo é uma continuidade do primeiro.

Vários dos fatores já mencionados podem ajudar a compreender que características pessoais, do ambiente, da cultura podem explicar de certa forma o uso da punição corporal, ou ao menos a posição favorável ao uso dela. Além disso, deve-se acrescentar um fator que não foi citado em nenhuma das 90 pesquisas analisadas: o efeito imediato da palmada. Este fator é muito importante, pois ele reforça e mantém o comportamento dos pais de baterem nos filhos. Por essa razão, compreende-se que o único efeito positivo encontrado por Gershoff (2002) e Larzelere (2000) foi a obediência imediata. É muito mais provável que se obtenha consequiências imediatas apresentando um estimulo punitivo do que apresentando um reforçador (Skinner, 1976; Sidman, 2001), "e isso significa que uma pessoa, provavelmente, acha mais fácil aprender técnicas de controle aversivo do que técnicas de reforço. No entanto, isso não significa que as técnicas aversivas sejam melhores" (Sidman, 2001, p. 128).

Além de as pessoas utilizarem esta prática por ela surtir efeito momentâneo, há uma forte influência da experiência prévia com a punição corporal, ou seja, pessoas que apanharam quando crianças são mais favoráveis ao uso deste método (Graziano \& Namste, 1990; Graziano \& cols., 1992; Rabin, 1997). A categoria 5 mostrou a tendência de se repetir a punição corporal de uma geração para outra. Pode-se compreender este fenômeno pelo aprendizado por imitação ou vicariante, ou seja, o indivíduo passou pela contingência e aprendeu que punição corporal funciona.

\section{Perpetuando um padrão de comportamento agressivo}

Aproveitando a discussão sobre transmissão intergeneracional da punição corporal, pode-se avaliar se isso é positivo ou negativo. Em primeiro lugar, é contraditório que pais e mães utilizem esta prática para punir comportamentos agressivos de seus filhos, como foi visto na pesquisa de Holden e cols. (1995). Em segundo lugar, mesmo que os pais batam em seus filhos por outros comportamentos inadequados, eles estão passando o modelo de agressividade para resolver um problema. Assim, crianças que apanham são mais agressivas com seus colegas (Strassberg \& cols., 1994); se envolvem mais em brigas (Simons \& cols., 1998); podem ser mais cruéis com animais na infância ou na adolescência (Flynn, 1999) e mais tarde podem ser mais agressivos com seus parceiros, chegando ao ponto de usar de violência contra a esposa (Good, 1999).

A evidência de que as pessoas que apanharam são mais agressivas demonstra que os pais, ao baterem, ensinam seus filhos que o comportamento agressivo é válido. Portanto, a criança poderá utilizar-se da agressão física em outras circunstâncias, nas quais ela queira estar no controle de alguém ou de alguma situação, ou seja, estará agindo de forma coercitiva de acordo com o modelo aprendido. A probabilidade de a criança seguir o modelo coercitivo aumenta quando ocorre uma associação entre a dor que a criança sente e o amor em relação aos seus pais. Geralmente a punição física é acompanhada por um discurso dos pais que amam a criança e que batem para o bem dela (Cornet, 1997). Além disso, a criança aprende a suportar situações aversivas e disfuncionais porque estão emparelhadas com situações de carinho e afeto (Weber, 2001).

\section{Efeitos negativos da punição corporal}

Além de a punição corporal estar inserida num ciclo de agressividade que se perpetua, ela traz outras conseqüências negativas para o desenvolvimento dos filhos. Straus (1994) sustenta que a punição corporal traz diversos prejuízos, o que pode ser verificado na análise da categoria 4. Certamente deve-se levar em conta que as pesquisas citadas possuem variedade metodológica para medir as variáveis dependente e independente, mas de uma forma geral, pode-se ter uma idéia de que a maioria das pesquisas encontraram que crianças e adolescentes que apanham em casa sofrem consequiências afetivas, emocionais, cognitivas e comportamentais. Nenhuma pesquisa trouxe fatores positivos.

Gershoff (2002) realizou uma revisão de literatura, encontrou apenas a obediência imediata como conseqüência positiva, já discutido anteriormente, e onze comportamentos e experiências indesejáveis associados à punição corporal, como: aumento da agressividade, aumento de comportamento delinqüente e antisocial, prejuízo da relação entre pais e filhos, prejuízo na saúde mental, aumento do risco de maltrato de filhos ou esposa.

Tanto Gershoff (2002) quanto Parke (2002) ressaltam a importância de se localizar a prática da punição corporal num sistema familiar composto por diversas outras práticas e estilos parentais. Essa preocu- 
pação apareceu em algumas pesquisas analisadas na categoria 4, que junto com a prática da punição corporal também avaliou outro aspecto do comportamento dos pais, como apoio emocional e envolvimento. Os problemas de comportamento dos filhos associados ao uso da punição corporal aumentaram no contexto de pouco apoio emocional (McLoyd \& Smith, 2002) e diminuíram com a presença do envolvimento parental (Simons \& cols., 1994).

A pesquisa longitudinal de Baumrind (2001) mostrou que, se os pais forem muito competentes e equilibrados em níveis de exigência e responsividade, uma palmada ocasional não causa dano terrível. Entretanto, apenas cerca de $35 \%$ da população tem o estilo parental mais adequado (Weber, Prado, Viezzer, Brandenburg, 2004), o participativo que combina exigência e responsividade, ou seja, uma minoria das crianças apanham num contexto familiar que pode atenuar prejuízos.

Larzelere (2000) defende que uma punição corporal aplicada por pais amorosos e com certos cuidados é efetiva. Tais cuidados são: a idade da criança de ser de dois a seis anos, deve ser usada com privacidade, sem exagero e sem perda de controle, acompanhada de diálogo, usada após um aviso prévio, havendo flexibilidade, motivada por interesse centrado na criança e não nos pais.

$\mathrm{O}$ ambiente familiar positivo, com pais competentes, talvez amenize os efeitos da punição corporal. Pode ser que uma minoria de pais tenha condições para seguir os passos sugeridos por Larzelere (2000). No entanto, os cuidados na aplicação da punição corporal e a afetividade dos pais não eliminam o fato de eles estarem transmitindo um modelo agressivo de resolver problemas, um modelo onde a força bruta de uma autoridade é justificável para o bem do outro, e não elimina o risco de a punição corporal se tornar um maltrato. A educação coercitiva traz, sim, prejuízos para o repertório do indivíduo (Sidman, 1995), efeitos colaterais não intencionados decorrem do uso da punição corporal e nem sempre são avaliados nas pesquisas.

\section{É possível mudar?}

Os pontos já levantados demonstraram como a punição corporal é fortemente controlada por regras e por contingências. Já nos anos 40, Rich (1946) afirmou que a punição corporal é uma tradição muito forte, cujo uso é motivado pela família e só pode ser modificado através da educação. Pesquisas analisadas na categoria 7 demonstraram como treinamentos educativos podem mudar a posição dos pais para menor apoio ao uso da punição corporal e para o menor uso desta prática (Rabin, 1997; Hopkins, 1998; Nicholson $\&$ cols., 2002). Estes treinamentos educativos podem ser mais efetivos quando se tem noção do que leva pais e mães a baterem em seus filhos (Ateah, 2001).

\section{CONCLUSÕES}

A presente pesquisa documental traz algumas contribuições importantes. Primeiro, pode-se perceber a multiplicidade de assuntos dentro do tema punição corporal, abrindo possibilidades diversas para a realização de pesquisas. Outra contribuição foi a avaliação de aspectos a favor e contra o uso de punição corporal. Em razão de tantos argumentos contra a palmada, a presente pesquisa vem trazer mais subsídios científicos para ser possível defender e proteger a criança contra qualquer tipo de agressão a ela aplicada, até mesmo a palmada que é comumente considerada "justificável" em nome da educação e da disciplina.

Na presente pesquisa foi estudada a punição corporal no contexto disciplinar, os pais bateram visando o bem dos filhos. Quando se compreende as opiniões dos pais e filhos, os fatores preditivos, a transmissão intergeneracional, entende-se os pais sem culpá-los. Os pais seguem regras e padrões de comportamentos aprendidos em sua própria história de vida. Cabe aos cientistas informar e mostrar para eles os pontos negativos da prática de punição corporal para eles poderem fazer escolha consciente de usar ou não. Isso tem sido feito em programas educativos para pais e tem gerado resultados positivos; os pais deixam de utilizar esta prática.

\section{REFERÊNCIAS}

Andero, A. A. \& Stewart, A. (2002). Issue of corporal punishment: re-examined. Journal of Instructional Psychology, 29(2), 90-96.

Ashton, V. (2001). The relationship between attitudes toward corporal punishment and the perception and reporting of child maltreatment. Child Abuse and Neglect, 25(3), 389-399.

Ateah, C. A. (2001). Determinants of maternal use of physical punishment: implications for child abuse prevention. Dissertation Abstracts International: Section B: The Sciences and Engineering, 61(7-B), 3535

Ateah, C. A. \& Parking, C. M. (2002). Childhood experiences with, and current attitudes toward, corporal punishment. Canadian Journal of Community Mental Health, 21(1), 35-46.

Bachar, E., Canetti, L., Bonne, O., Denour, A. K. \& Shalev, A.Y (1997). Physical punishment and signs of mental distress in normal adolescents. Adolescence, 32(128), 945-958.

Barrish, B. M. (1996). The relationship of remembered parental physical punishment to adolescent self-concept. Dissertation Abstracts International: Section B: The Sciences and Engineering, 57(3-B), 2171.

Baumrind, D. (2001). Does relevant research support a blanket injunction against disciplinary spanking by parents? Em $109^{\text {a }}$ Annual Convention of American Psychological Association. Obtido em 29 de abril de 2002 do World Wide Web: http://ihd.berkeley.edu/.

Blampied, N. M. \& Kahan, E. (1992). Acceptability of alternative punishments: a community survey. Behavior Modification, 16(3), 400-413. 
Bradley, R. H., Corwyn, R. F., Burchinal, M., Pipes-Mcadoo, H \& Garcia-Coll, C. (2001). The home environments of children in the United States. Part II: Relations with behavioral development through age thirteen. Child Development, 72(6), 18681886.

Camargo, C. L. (1996). Violência física contra crianças e adolescentes: um recorte localizado. Tese de Doutorado, Faculdade de Saúde Pública, Universidade de São Paulo. São Paulo, SP.

Carswell, W. A. (1990). Perfil de um grupo de pais perpetradores de maus-tratos e dos filhos - suas vítimas. Tese de Doutorado, Escola de Enfermagem de Ribeirão Preto, Universidade de São Paulo. Ribeirão Preto, SP.

Catron, T. F. \& Masters, J. C. (1993). Mothers' and children's conceptualizations of corporal punishment. Child Development, 64(6), 1815-1828.

Cherian, V. I. (1994). Self-reports of corporal punishment by Xhosa children from broken and intact families and their academic achievement. Psychological Reports, 74(3, Pt 1), 867874.

Christensen, E. (2001). Brug af legemlig afstraffelse i opdragelsen af 3-arige. / The use of corporal punishment among three-yearolds. Nordisk Psykologi, 53(1), 47-71.

Cornet, J. (1997). Faut-il battre les enfants? Paris: Hommes et Perspectives.

Crouch, J. L. \& Behl, L. E. (2001). Relationships among parental beliefs in corporal punishment, reported stress, and physical child abuse potential. Child Abuse and Neglect, 25(3), 413-419.

Culp, R. E., Culp, A. M., Dengler, B. \& Maisano, P. C. (1999). First-time young mothers living in rural communities using corporal punishment with their toddlers. Journal of Community Psychology, 27(4), 503-509.

Davis, P. W. (1999). Corporal punishment cessation: social contexts and parents' experiences. Journal of Interpersonal Violence, 14(5), 492-510.

Devet, K. A. (1997). Parent-adolescent relationships, physical disciplinary history, and adjustment in adolescents. Family Process, 36(3), 311-322.

Dietz, T. L. (2000). Disciplining children: characteristics associated with the use of corporal punishment. Child Abuse and Neglect, 24(12), 1529-1542.

Durrant, J. E. (2000). Trends in youth crime and well-being since the abolition of corporal punishment in Sweden. Youth and Society, 31(4), 437-455.

Durrant, J. E., Broberg, A. G. \& Rose, K. L. (1999). Predicting mother's use of physical punishment during mother-child conflicts in Sweden and Canada. Psychological Reports, 85(2), 381-392.

Eamon, M. K. \& Zuehl, R. M. (2001). Maternal depression and physical punishment as mediators of the effect of poverty on socioemotional problems of children in single-mother families. American Journal of Orthopsychiatry, 71(2), 218-226.

Ellison, C. G. \& Sherkat, D. E. (1993). Conservative Protestantism and support for corporal punishment. American Sociological Review, 58(1), 131-144.

Ellison, C. G., Bartkowski, J. P. \& Segal, M. L. (1996). Do conservative Protestant parents spank more often? Further evidence from the National Survey of Families and Households. Social Science Quarterly, 77(3), 663-673.

Englehart, R. J. \& Hale, D. B. (1990). Punishment, nail-biting, and nightmares: a cross-cultural study. Journal of Multicultural Counseling and Development, 18(3), 126-132.

Flynn, C. P. (1999). Animal abuse in childhood and later support for interpersonal violence in families. Society and Animals, $7(2), 161-172$
Flynn, C. P. (1994). Regional differences in attitudes toward corporal punishment. Journal of Marriage and the Family, 56(2), 314-324.

Flynn, C. P. (1996). Regional differences in spanking experiences and attitudes: a comparison of northeastern and southern college students. Journal of Family Violence, 11(1), 59-80.

Flynn, C. P. (1998). To spank or not to spank: The effect of situation and age of child on support for corporal punishment. Journal of Family Violence, 13(1), 21-37.

Frias-Armenta, M. (1999). Law, psychology, family relations and child abuse in Mexico. Abstracts International: Section B: The Sciences and Engineering, 60(4-B), 1913.

Frias-Armenta, M. (2002). Long-term effects of child punishment on Mexican women: a structural model. Child Abuse and Neglect, 26(4), 371-386.

Furlotti, T. V. M. (1999). Segredos de família: violência doméstica contra crianças e adolescentes na São Paulo das primeiras décadas do século XX. Dissertação de Mestrado, Faculdade de Filosofia, Letras e Ciência Humanas, Universidade de São Paulo. São Paulo, SP.

Furnham, A. (1995). Attitudes to spanking children. Personality and Individual Differences, 19(3), 397-398.

Gershoff, E. T. (2002). Corporal punishment by parents and associated child behaviors and experiences: A meta-analytic and theoretical review. Psychological Bulletin, 128(4), 539-579.

Gershoff, E. T., Miller, P. C. \& Holden, G. W. (1999). Parenting influences from the pulpit: religious affiliation as a determinant of parental corporal punishment. Journal of Family Psychology, 13(3), 307-320.

Giles-S., J., Straus, M. A.\& Sugarman, D. B. (1995). Child, maternal, and family characteristics associated with spanking. Family Relations: Journal of Applied Family and Child Studies, 44(2), 170-176.

Good, J. A. (1999). Shame, images of God and the cycle of violence in adults who experienced childhood corporal punishment. Dissertation Abstracts International Section A: Humanities and Social Sciences, 59(11-A), 4292.

Gopaul-Mcnicol, S. A. (1999). Ethnocultural perspectives on childrearing practices in the Caribbean. International Social Work, 42(1), 79-86.

Gough, B. \& Reavey, P. (1997). Parental accounts regarding the physical punishment of children: discourses of dis/empowerment. Child Abuse and Neglect, 21(5), 417-430.

Grasmick, H. G., Bursik, R. J. \& Kimpel, M. (1991). Protestant fundamentalism and attitudes toward corporal punishment of children. Violence and Victims, 6(4), 283-298.

Graziano, A. M. \& Namaste, K. A. (1990). Parental use of physical force in child discipline: a survey of 679 college students. Journal of Interpersonal Violence, 5(4), 449-463.

Graziano, A. M., Lindquist, C. M., Kunce, L. J. \& Munjal, K. (1992). Physical punishment in childhood and current attitudes: an exploratory comparison of college students in the United States and India. Journal of Interpersonal Violence, 7(2), 147155.

Holden, G. W., Coleman, S. M. \& Schmidt, K. L. (1995). Why 3year-old children get spanked: parent and child determinants as reported by college-educated mothers. Merrill Palmer Quarterly, 41(4), 431-452.

Holden, G. W., Thompson, E. E., Zambarano, R. J. \& Marshall, L. A. (1997). Child effects as a source of change in maternal attitudes toward corporal punishment. Journal of Social and Personal Relationships, 14(4), 481-490.

Hopkins, K. (1998). A descriptive study of African-American professionals' attitudes toward corporal punishment. Dissertation Abstracts International: Section B: The Sciences and Engineering, 58(10-B), 5633.

Jackson, A. P., Gyamfi, P., Brooks, G. J. \& Blake, M. (1998). Employment status, psychological well-being, social support, 
and physical discipline practices of single Black mothers. Journal of Marriage and the Family, 60(4), 894-902.

Kaplan, J. P. (1996). Psychologists' attitudes towards corporal punishment. Dissertation Abstracts International: Section B: The Sciences and Engineering, 56(9-B), p. 5151.

Kelder, L. R., Mcnamara, J. R., Carlson, B. \& Lynn, S. J. (1991) Perceptions of physical punishment: The relation to childhood and adolescent experiences. Journal of Interpersonal Violence, 6(4), 432-445.

Larzelere, R. E. (2000). Child outcomes of nonabusive and customary physical punishment by parents: An updated literature review. Clinical Child and Family Psychology Review. 3(4), 199-221.

Larzelere, R. E. \& Johnson, B. (1999). Evaluations of the effects if Sweden's spanking ban on physical child abuse rates: a literature review. Psychological Reports, 85(2), 381-392.

Longo. C. S., Azevedo M. A \& Guerra, V. N. A. (2002). Bibliografia Seletiva (Nacional e Internacional) sobre Punição Corporal Doméstica de Crianças e Adolescentes. Instituto de Psicologia: USP.

Mardis, Y. S. (2001). Parental attitudes and attitudes toward spanking: A survey of social workers' attitudes. Dissertation Abstracts International Section A: Humanities and Social Sciences, 62(2-A), 776.

Matta, M. A. (2002). Parental corporal punishment as a predictor of child maladjustment: race and parental responsiveness as potential moderators. Dissertation Abstracts International: Section B: The Sciences and Engineering, 63(4-B), 2091.

Mcloyd, V. C.\& Smith, J. (2002). Physical discipline and behavior problems in African American, European American and Hispanic children: emotional support as a moderator. Journal of Marriage and Family, 64(1), 40-53.

Meneguel, S. N., Giugliani, E. J. \& Falceto, O. (1998). Relações entre violência doméstica e agressividade na adolescência. Cadernos de Saúde Pública, 14(2), 327-358.

Morris, J. D. (1996). The normative influence of social network members on mothers' attitudes toward corporal punishment. Dissertation Abstracts International: Section B: The Sciences and Engineering, 57(1-B), 765.

Mosby, L., Rawls, A. W., Meehan, A. J., Mays, E. \& Pettinari, C. J. (1999). Troubles in interracial talk about discipline: an examination of African American child rearing narratives. Journal of Comparative Family Studies, 30(3), 489-521.

Muller, R. T. (1996). Family aggressiveness factors in the prediction of corporal punishment: reciprocal effects and the impact of observer perspective. Journal of Family Psychology, 10(4), 474-489.

Muller, R. T., Hunter, J. E. \& Stollak, G. (1995). The intergenerational transmission of corporal punishment: a comparison of social learning and temperament models. Child Abuse and Neglect, 19(11), 1323-1335.

Murphy-Cowan, T \& Stringer, M. (1999). Physical punishment and the parenting cycle: A survey of Northern Irish parents. Journal of Community and Applied Social Psychology, 9(1), 61-71.

Nicholson, B., Anderson, M., Fox, R. \& Brenner, V. (2002). One family at a time: a prevention program for at-risk parents. Journal of Counseling and Development, 80(3), 362-371.

Nobes, G., Smith, M., Upton, P. \& Heverin, A. (1999). Physical punishment by mothers and fathers in British homes. Journal of Interpersonal Violence, 14(8), 887-902.

Nobes, G. \& Smith, M. (2002). Family structure and the physical punishment of children. Journal of Family Issues, 23(3), 349373.

Nobes, G. \& Smith, M. (1997). Physical punishment of children in two-parent families. Clinical Child Psychology and Psychiatry, 2(2), 271-281.
Parke, R. (2002). Punishment revisited-Science, values, and the right question: comment on gershoff (2002). Psychological Bulletin. 128(4), 596-601.

Pascolat, G. \& cols. (2001). Abuso físico: o perfil do agressor e da criança vitimizada. Jornal de Pediatria, 77(1), 35-40.

Rabin, B. E. (1997). Changing medical students' attitudes toward corporal punishment and knowledge of alternatives via an educational intervention. Dissertation Abstracts International: Section B: The Sciences and Engineering, 58(6-B), 3305.

Rich, G. J. (1946). The tradition of force and punishment. Nervous Child, 5, 222-225.

Rohner, R. P.; Kean, K. J. \& Cournoyer, D. E. (1991). Effects of corporal punishment, perceived caretaker warmth, and cultural beliefs on the psychological adjustment of children in St. Kitts, West Indies. Journal of Marriage and the Family, 53(3), 681693.

Roque, E. M. S. (2001). A violência na família contra crianças e adolescentes e a percepção dos operadores de direito, na comarca da Jardinópolis - SP. Dissertação de Mestrado, Escola de Enfermagem de Ribeirão Preto, Universidade de São Paulo. Ribeirão Preto.

Saffer, V. J. (1999). Discipline: an interactive model. Dissertation Abstracts International Section B: The Sciences and Engineering, 60(3-B), 1314.

Santos, E. V. (1999). Para além do desfecho: a violência física doméstica contra o escolar como processo social e como questão de saúde coletiva. Tese de Doutorado, Escola de Enfermagem, Universidade de São Paulo, São Paulo.

Seligman, M. E. P. (1977). Desamparo: sobre depressão, desenvolvimento e morte. São Paulo: Hucitec/EDUSP.

Sherman, R. (1997). A study of factors relating to the intergenerational cycle of corporal punishment in parents whose children attend nonpublic school. Dissertation Abstracts International Section A: Humanities and Social Sciences, 58(6-A), 2074.

Shor, R. (1999). Beyond a cross-cultural definition of child maltreatment: comparing immigrants from the Caucasus and European countries of the former Soviet Union. Early Child Development and Care, 150, 1-15.

Sidman, M. (2001). Coerção e suas implicações. Campinas: Livro Pleno.

Simons, R. L., Lin, K. H., Gordon, L. C., Brody, G. H., Murry, V. \& Conger, R. D. (2002). Community differences in the association between parenting practices and child conduct problems. Journal of Marriage and Family, 64(2), 331-345.

Simons, R. L., Johnson, C. \& Conger, R. D. (1994). Harsh corporal punishment versus quality of parental involvement as an explanation of adolescent maladjustment. Journal of Marriage and the Family, 56(3), 591-607.

Simons, R. L., Lin, K. H. \& Gordon, L. C. (1998). Socialization in the family of origin and male dating violence: A prospective study. Journal of Marriage and the Family, 60(2), 467-478.

Skinner, B. F. (1976). Ciência e comportamento humano. São Paulo: EDART.

Spencer, M. J. (1999). Corporal punishment and ridicule: residual psychological effects in early adulthood. Implications for counselors. Dissertation Abstracts International Section A: $\mathrm{Hu}$ manities and Social Sciences, 60(4-A), p. 1030.

Stolley, K. S. \& Szinovacz, M. (1997). Caregiving responsibilities and child spanking. Journal of Family Violence, 12(1), 99-112.

Strassberg, Z., Dodge, K. A., Pettit, G. S. \& Bates, J. E. (1994). Spanking in the home and children's subsequent aggression toward kindergarten peers. Development and Psychopathology, 6(3), 445-461.

Straus, M. A \& Kantor, G. K. (1994). Corporal punishment of adolescents by parents: a risk factor in the epidemiology of de- 
pression, suicide, alcohol abuse, child abuse, and wife beating. Adolescence, 29(115), 543-561.

Straus, M. A. \& Stewart, J. H. (1999). Corporal punishment by American parents: National data on prevalence, chronicity, severity, and duration, in relation to child and family characteristics. Clinical Child and Family Psychology Review, 2(2), $55-70$.

Straus, M. A. (1994). Should the use of corporal punishment by parents be considered child abuse? Yes. Em M. A. Manson. \& E. Gambrill (Orgs.), Debating children's lives: current controversies on children and adolescents. Thousand Oaks, CA: Sage Publications.

Straus, M. A. (2000). Corporal punishment and primary prevention of physical abuse. Child Abuse and Neglect, 24(9), 11091114.

Straus, M. A. \& Donnelly, D. A. (1993). Corporal punishment of adolescents by American parents. Youth and Society, 24(4), 419-442.

Straus, M. A. \& Mouradian, V. E. (1998). Impulsive corporal punishment by mothers and antisocial behavior and impulsiveness of children. Behavioral Sciences and the Law, 16(3), 353374.

Taleb, T. A. (2001). The prevalence of physical punishment choice among mothers of kindergarten children, the types of behaviors identified as deserving punishment and the relationship between punishment, and children's behavioral characteristics. Dirasat: Educational Sciences, 28(1), 229-246.

Tschoban, M. (2001). A psychophysiological investigation of observed corporal punishment as a stressor in parents. Dissertation Abstracts International: Section B: The Sciences and Engineering, 61(10-B), p. 5613.

Turner, H. A. \& Finkelhor, D. (1996). Corporal punishment as a stressor among youth. Journal of Marriage and the Family, 58(1), 155-166.

Vargas, N. A.; Lopez, D.; Perez, P. \& Zuniga, P. (1995). Parental attitude and practice regarding physical punishment of school children in Santiago de Chile. Child Abuse and Neglect, 19(9), 1077-1082.

Wade, K. B. (2000). Acculturation processes, psycho-social resources, and child discipline: a comparison of Latina and EuroAmerican mothers. Dissertation Abstracts International: Section B: The Sciences and Engineering, 61(4-B), p. 2277.
Walsh, W. (2002). Spankers and non spankers: Where they get information on spanking. Family Relations: Interdisciplinary Journal of Applied Family Studies, 51(1), 81-88.

Watson, C. D. (2002). The Parental Anger Scale-3 (PAS-3): reliability and validity using a sample from the World Wide Web. Dissertation Abstracts International: Section B: The Sciences and Engineering, 62(10-B), 4809.

Weber, L. N. D. (2001). Quem ensina a violência? Conjuntura Social, 6, 38-43.

Weber, L. N. D., Viezzer, A. P., Brandenburg, O. J. \& Zocche, C. R. E. (2002). Famílias que maltratam: uma tentativa de socialização pela violência. Psico-USF, 7, 157-167.

Weber, L. N. D., Prado, P. M., Viezzer, A. P. \& Brandenburg, O. J. (2004). Identificação de estilos parentais: o ponto de vista dos pais e dos filhos. Psicologia Reflexão e Crítica, 17(3), 323331.

Weber, L. N. D., Viezzer, A. P. \& Brandenburg, O. J. (2004). O uso de surras e palmadas como prática educativa. Estudos de Psicologi (Natal), 9(2), 227-238.

Weitz, A. (2001). An investigation of the relationship between observing corporal punishment and childhood stress. Dissertation Abstracts International: Section B: The Sciences and Engineering, 61(10-B), p. 5614.

Wiehe, V. R. (1990). Religious influence on parental attitudes toward the use of corporal punishment. Journal of Family Violence, 5(,173-186.

Wissow, L. S. (2001). Ethnicity, income, and parenting contexts of physical punishment in a national sample of families with young children. Child Maltreatment: Journal of the American Professional Society on the Abuse of Children, 6(2), 118-129.

Woodward, L. J.\& Fergusson, D. M. (2002). Parent, child and contextual predictors of childhood physical punishment. Infant and Child Development, 11, 213-236.

Yousser, R. M., Attia, M. S. E. \& Kamel, M. I. (1998). Children experiencing violence I: Parental use of corporal punishment. Child Abuse and Neglect, 22(10), 959-973.

Enviado: $10 / 04 / 2005$ Revisado: 20/05/2005 Aceito: $30 / 05 / 2005$

\section{Sobre as autoras:}

Olivia Justen Brandenburg: Psicóloga, voluntária do Projeto Criança: Desenvolvimento, Educação e Cidadania, coordenado pela Prof ${ }^{a}$. Lidia Natalia Dobrianskyj Weber, da Universidade Federal do Paraná.

Lidia Natalia Dobrianskyj Weber: Universidade Federal do Paraná. Endereço para correspondência: UFPR - Departamento de Psicologia - Caixa Postal 19.061 - 80060-000 Curitiba-PR. Endereço eletrônico: lidiaw@uol.com.br. 